\title{
Epidemiology and Retrospective Analysis in Extrapulmonary Neuroendocrine Carcinoma
}

\author{
Ashish Sethi ${ }^{1}$, Mohammad Islam $^{1}$, Raj Moses ${ }^{1}$, Gene Finley ${ }^{1}$ \\ 1. Hematology and Medical Oncology, Allegheny Health Network, Pittsburgh, USA
}

Corresponding author: Ashish Sethi, sethi_ash97@rediffmail.com

\begin{abstract}
Objectives: We conducted a retrospective analysis of patients with extrapulmonary neuroendocrine carcinomas (EPNECs) to explore the distribution and overall outcomes by different regimens and their primary sites.
\end{abstract}

Setting: We reviewed the outcomes of one of the largest data sets of patients with extrapulmonary small cell carcinomas (EPSCCs) identified at Allegheny General Hospital located in Pittsburgh, Pennsylvania, USA.

Participants: Patients diagnosed with grade 3 EPNECs were retrospectively identified.

Primary endpoint and epidemiology: Overall survival (OS) with different treatment regimens was the primary endpoint. Also, epidemiological factors such as risk factors, race, family history of cancer, and associated comorbidities were recorded.

Results: OS was 16 months in seven patients who received cisplatin/etoposide chemotherapy and 8.5 months in seven patients with carboplatin/etoposide chemotherapy. The commonest primary site was the gastrointestinal tract (GIT). Smoking history association was observed to be $50 \%$. Merkel cell carcinoma (MCC) patients had significantly better OS. Simultaneously, an extensive form of disease pattern was also noticed in $94.4 \%$ of the patients. Significantly, neutropenic sepsis was observed in $71.4 \%$ of the patients who were treated with cisplatin/etoposide combination.

Conclusion: EPNECs demonstrated a low response rate to chemotherapy and high rates of distant metastases. Conclusively, brain metastases were rare.

Review began 12/09/2020 Review ended 01/13/2021 Published 01/17/2021

(๑) Copyright 2021 Sethi et al. This is an open access article distributed under the terms of the Creative Commons Attribution License CC-BY 4.0., which permits unrestricted use, distribution, and reproduction in any medium, provided the original author and source are credited.
Categories: Internal Medicine, Gastroenterology, Oncology

Keywords: extrapulmonary neuroendocrine carcinoma (epnec), poorly differentiated neuroendocrine carcinoma (pdnec), grade 3 neuroendocrine carcinoma, neuroendocrine carcinoma (nec)

\section{Introduction}

Poorly differentiated neuroendocrine carcinomas (PDNECs) or high grade 3 type, also called small cell carcinomas (SCCs), is considered one of the most aggressive forms of malignancy arising from the epithelial tissues. Neuroendocrine carcinomas (NECs) can affect any organ system in the body, and most of these cases occur primarily in the lung. Extrapulmonary neuroendocrine carcinomas (EPNECs) refers to NECs occurring in any organ type except lung, viz gastrointestinal tract, genitourinary system, skin, bone, and pericardium. Only $5 \%$ of SCCs are extrapulmonary, with incidence rate ranging between $0.1 \%$ to $0.4 \%$ [1]. PDNECs account for as much as $10 \%$ to $20 \%$ of malignant digestive neuroendocrine neoplasms (NENs) [2]. Most EPNECs present with widespread metastatic disease at the time of diagnosis and have a poor prognosis with a median survival duration of 34 months in localized disease, 14 months in regional disease, and five months in distant disease [3].

Treatment such as chemotherapy with etoposide and platinum is considered the preferred (or standard) first-line treatment for both metastatic and localized NECs. Localized disease can also be treated surgically, if possible, or with chemo-radiation. It has also been noticed that the incidence of brain micrometastases in small cell lung cancer (SCLC) is high $\sim 10 \%$ in comparison to EPNEC. Thus, it necessitates prophylactic cranial irradiation in the SCLC.

\section{Materials And Methods}

We conducted a retrospective analysis of clinical outcomes in 18 patients diagnosed with grade 3 EPNECs between January 1, 2018, and May 27, 2020, at Allegheny General Hospital located in Pittsburgh, Pennsylvania, USA. The study received approval from the Internal Review Board. Medical data were extracted from the Epic system. Overall survival (OS) with different treatment regimens was the primary endpoint, and epidemiological factors such as risk factors, race, family history of cancer, and associated comorbidities were recorded. Grade 3 or PDNECs were delineated based on histological features with $>20$ mitoses per 10 high 
power field (HPF) or Ki-67 labeling index >20\%. Patients were divided into metastatic or non-metastatic disease based on clinical presentation.

\section{Results}

Of the 18 patients, $50 \%$ were male. Our results demonstrated a median age of 63 years. The youngest individual was 29 years, while the eldest one was 85 years of age. Nearly $94.4 \%$ of NECs were observed specifically in the Caucasian race, and the majority of the presentation at the time of diagnosis was metastatic in nature, accounting for $94.4 \%$.

A family history of cancer from our patient data was observed in $66.6 \%$ of cases. Another very likely causative factor was observed to be having a history of smoking, and the association was noticed in $50 \%$ of patients, whereas a history of alcohol consumption was noticeably present in $44.4 \%$ of the patients.

The most common location for EPNEC occurrence was observed in the gastrointestinal tract (GIT) in about $66.6 \%$ of patients. A noticeable involvement of the pancreas in GIT as a primary origin was maximum in $33.3 \%$ of patients. This was subsequently followed by the sigmoid colon in $25 \%$ of patients, and occurrence in the rectum was also recorded to be $25 \%$. Stomach and common bile duct as primary origin sites were observed in one patient each. One case was diagnosed as metastasis of unknown primary origin. Genitourinary tract involvement as EPNEC was observed in $16.6 \%$ of patients. Ovary, as the primary site of origin, was involved in $66.6 \%$ of patients. We also observed two patients diagnosed with Merkel cell carcinoma (MCC), one with localized and one with extensive disease; both responded well to treatment and remain alive for 15 months and 25 months, respectively.

The liver was observed to be the commonest metastatic site (88.8\%). One patient with extensive MCC was observed to have metastasis to bone and regional lymph nodes. Neutropenic sepsis was noticed in $71.4 \%$ of patients treated with the cisplatin-etoposide regimen in comparison to $28.5 \%$ with the carboplatinetoposide combination group.

The most common regimen used in our study was the combination of etoposide-carboplatin and etoposidecisplatin. OS was 16 months in seven patients who received cisplatin-etoposide chemotherapy and 8.5 months in seven patients with carboplatin-etoposide chemotherapy. Although the OS with cisplatinetoposide was numerically better than carboplatin-etoposide, this was not statistically significant (pvalue $=0.29$ ). Progression free survival (PFS) of 27 months with etoposide-carboplatin in one patient categorized as NEC of the ovary was seen. One patient in our study with NEC of the common bile duct received etoposide/carboplatin and atezolizumab combination based on IMpower133 trial with PFS of four months.

Pertinent findings regarding epidemiology, disease pattern, and treatment response are summarised in Tables 1-3. 


\section{Cureus}

\begin{tabular}{|c|c|c|}
\hline Epidemiology Factors and Disease pattern & No. of Patients & Percentages (\%) \\
\hline Male Gender & 9 & 50 \\
\hline Female Gender & 9 & 50 \\
\hline Caucasian Race & 17 & 94.4 \\
\hline Family History & 12 & 66.6 \\
\hline Smoking History & 9 & 50 \\
\hline Alcohol consumption & 8 & 44.4 \\
\hline Metastatic or Extensive in nature & 17 & 94.4 \\
\hline GIT Involvement & 12 & 66.6 \\
\hline Pancreas & 4 & 33.3 \\
\hline Sigmoid colon & 3 & 25 \\
\hline Rectum & 3 & 25 \\
\hline Genitourinary Tract Involvement & 3 & 16.6 \\
\hline Ovary & 2 & 66.6 \\
\hline Liver as Metastatic involvement & 16 & 88.8 \\
\hline Neutropenic Sepsis with Cisplatin/ttoposide & 5 & /1.4 \\
\hline Neutropenic Sepsis with Carboplatin/Etoposide & 2 & 28.5 \\
\hline
\end{tabular}

TABLE 1: Demographic Features and Clinical Presentation

GIT, Gastrointestinal Tract 


\section{Cureus}

\begin{tabular}{|llllll|}
\hline Age & Race & Sex & Location of Primary Tumor & Distant Metastasis & Overall Survival (OS) \\
\hline 65 & Caucasian & Male & Urinary bladder & Liver & 13 months \\
56 & African & Female & Pancreas & Liver & 3.5 months \\
53 & Caucasian & Male & Pancreas & Liver & 5 months \\
53 & Caucasian & Male & Rectum & Liver & 24 months \\
59 & Caucasian & Male & Pancreas & Liver & 7 months \\
29 & Caucasian & Female & Ovary & Liver & 7 months \\
51 & Caucasian & Female & Sigmoid colon & Liver & 10 months \\
62 & Caucasian & Male & Rectum & Liver & 5 months \\
77 & Caucasian & Female & Rectum & Liver & 1.5 months \\
56 & Caucasian & Male & Pancreas & Liver & 4 months \\
70 & Caucasian & Female & Sigmoid colon & Liver & 14 months \\
85 & Caucasian & Male & Sigmoid colon & Liver & 4 months \\
78 & African & Female & Unknown Primary & Liver & 33 months \\
64 & Caucasian & Male & Gastric & Liver & 48 months
\end{tabular}

\section{TABLE 2: OS and Epidemiology}

OS, Overall Survival: Length of time from either the date of diagnosis or the start of treatment for the disease.

\begin{tabular}{|c|c|c|c|c|c|}
\hline Age & Race & Sex & Location of Primary Tumor & Distant Metastasis & Progression Free Survival (PFS) \\
\hline 64 & Caucasian & Female & Ovary & Liver & 27 months \\
\hline 67 & Caucasian & Female & Ampulla of Vater/Common bile duct (CBD) & Liver & 4 months \\
\hline 66 & Caucasian & Male & Merkel cell carcinoma & NA & 15 months \\
\hline 54 & Caucasian & Female & Merkel cell carcinoma & $\begin{array}{l}\text { Bone, Distant lymph } \\
\text { node }\end{array}$ & 25 months \\
\hline
\end{tabular}

\section{TABLE 3: PFS and Epidemiology}

PFS, Progression Free Survival: The length of time during and after the treatment of a disease that a patient lives with the disease, but it does not get worse.

NA: Not Applicable

\section{Discussion}

NECs have been associated with hereditary syndromes such as Von Hippel Lindau syndrome, neurofibromatosis type 1, multiple endocrine neoplasia type 1 and 2 (MEN 1/MEN 2), and tuberous sclerosis complex; however, none of our patients had a suggestive history. Nonetheless, the family history of different cancer types was observed in $66 \%$ of cases in our study.

PDNECs and neuroendocrine tumors (NETs) are defined on the basis of expression of neuroendocrine markers such as chromogranin A and synaptophysin (Figure 1) with aggressive histologic features of PDNECs illustrating as high mitotic rate $>10$ mitotic figures/10 HPF, extensive necrosis, nuclear atypia, and the Ki-67 proliferation index, if performed, of more than $20 \%$ (Table 4 ) 


\section{Cureus}

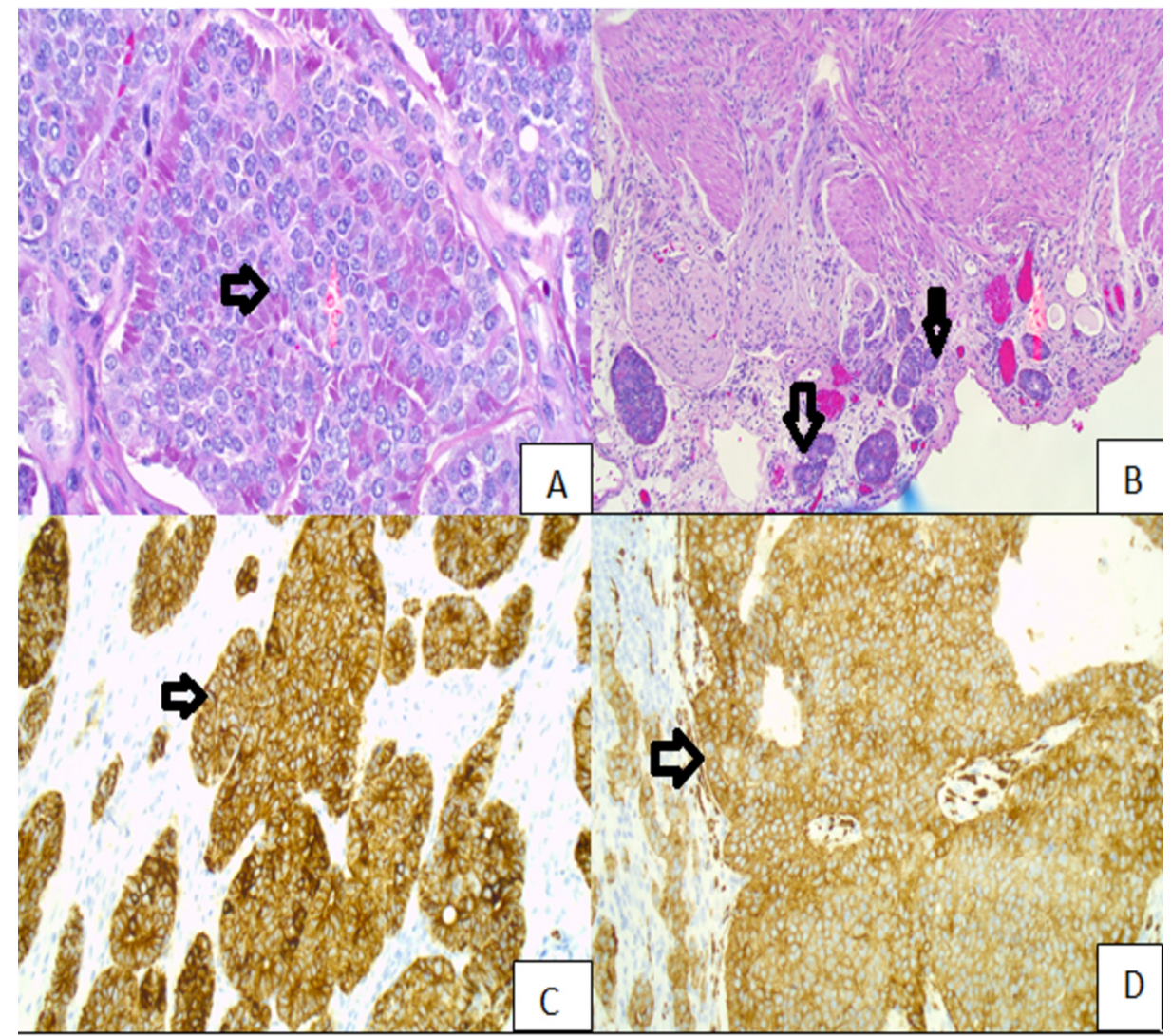

FIGURE 1: (A) NET. (B) NET invading visceral peritoneum. (C) Positive staining for pan-cytokeratin. (D) Positive staining for synaptophysin.

NET, Neuroendocrine tumor.

Permission was taken from the original publisher; adapted from Aslam et al.[4].

\begin{tabular}{|c|c|c|c|}
\hline Neuroendocrine Neoplasm & Morphology (Differentiation) & Grading G1-G3 (Ki-67 index in \%) & Abbreviation \\
\hline Neuroendocrine tumor Grade 1 & Well-differentiated & G1 ( $\leq 2 \%)$ & NET G1 \\
\hline Neuroendocrine tumor Grade 2 & Well-differentiated & G2 (3-20\%) & NET G2 \\
\hline Neuroendocrine tumor Grade 3 & Well-differentiated & G3 (>20\%) & NET G3 \\
\hline Neuroendocrıne carcınoma & Poorly-differentıated (large or small cell) & Gi3 (>20\%) & NEC \\
\hline
\end{tabular}

\section{TABLE 4: Neuroendocrine Morphology and Grade Classification}

NENs grade 3 represents two groups regarding the morphology and the grading: NECs and neuroendocrine tumor grade 3 (NET G3) [5].

Among 18 patients, $50 \%$ of the patients were men (nine patients), and out of them, approximately $60 \%$ of the affected patients had a history of smoking. Kim et al. have suggested no correlation between smoking and extrapulmonary small cell carcinomas (EPSCCs) [6].

In a similar study, focusing on primary NETs arising from the hepatobiliary and pancreatic regions, a response rate of $14 \%$ and median survival of 5.8 months were obtained in response to cisplatin plus etoposide [7]. In patients manifesting progressive disease after platinum-based chemotherapy, no standard alternate treatment is available [8]. Compared to the studies mentioned above, the average median OS in our patients was 8.5 months and 16 months for etoposide-carboplatin and etoposide-cisplatin, respectively.

Further, in contrast to SCLC, topotecan as a second-line treatment option did not induce any remissions and 
New advances in the management of small cell lung cancer with immunotherapy have shown great promise in recent clinical trials, such as the CASPIAN trial, which studied the effect of adding durvalumab to the standard therapy in patients with extensive stage small cell lung carcinoma (ES-SCLC) [10]. Durvalumab plus platinum-etoposide was associated with a significant improvement in overall survival, with a hazard ratio of 0.73 (95\% CI, $0 \cdot 59-0 \cdot 91 ; \mathrm{p}=0.0047)$ and median overall survival of 13.0 months $(95 \% \mathrm{CI}, 11 \cdot 5-14 \cdot 8)$ in the durvalumab plus platinum-etoposide group versus $10 \cdot 3$ months (95\% CI, 9.3-11.2) in the platinumetoposide group [10]. IMpower133 trial, which studied the use of azetolizumab in ES-SCLC, revealed longer OS in the atezolizumab group (median, 12.3 months; 95\% CI, 10.8-15.9) than in the placebo group (median, 10.3 months; 95\% CI, 9.3-11.3) [11].

Only one patient in our study with NEC of the common bile duct received IMpower133 and remains without progression four months into first-line therapy. None of the patients from our data list were enrolled for the CASPIAN trial as first-line therapy for EPNEC, and none of them received cranial irradiation during the treatment course.

Lastly, MCC, which also represents a high-grade EPNEC, is an aggressive form of skin carcinoma which can be localized or metastatic at the time of presentation. Surgical excision, chemo-radiation, and immunotherapy are considered as different management approaches for this subtype based on clinical presentation. However, a study done on advanced MCC resulted in an objective response rate (RR) of 56\% with pembrolizumab as first-line treatment [12]. Prognosis of MCC is often poor and is dependent on the stage at presentation.

A number of biases may have influenced our results given the limited number of patients involved and lack of central pathology verification; we still observed certain pertinent inferences from our study. It was noticed that the association of smoking was relatively less compared to SCLC. Also, none of our patients had cerebral metastasis on magnetic resonance imaging (MRI). The pancreas as the primary site of origin was considered the most aggressive form of NEC, signifying poor prognosis with OS ranging between 3.5 months and seven months (Table 2).

\section{Conclusions}

EPNECs is a rare entity with a variable response to platinum and etoposide. In our case series, the median survival was approximately one year. Cisplatin provided additional benefit at the cost of increased toxicity, mainly in the form of gastrointestinal toxicities and neutropenic sepsis. Also, many patients with comorbidities or advanced age cannot tolerate cisplatin. The results of platinum-based chemotherapy are unsatisfactory, and clearly, new approaches are needed. Promising results in small cell lung cancer reported in the IMpower133 and CASPIAN trials may be a path forward. More research is needed to define the role of immunotherapy in EPNEC, expanding treatment options for both patients and physicians.

\section{Additional Information \\ Disclosures}

Human subjects: All authors have confirmed that this study did not involve human participants or tissue. Animal subjects: All authors have confirmed that this study did not involve animal subjects or tissue. Conflicts of interest: In compliance with the ICMJE uniform disclosure form, all authors declare the following: Payment/services info: All authors have declared that no financial support was received from any organization for the submitted work. Financial relationships: All authors have declared that they have no financial relationships at present or within the previous three years with any organizations that might have an interest in the submitted work. Other relationships: All authors have declared that there are no other relationships or activities that could appear to have influenced the submitted work.

\section{Acknowledgements}

The completion of this undertaking could not have been possible without the participation and assistance of so many people whose names may not all be enumerated. Their contributions are sincerely appreciated and gratefully acknowledged. To all relatives, friends, and others who in one way or another shared their support, either morally, financially, and physically, thank you.

\section{References}

1. Wong YN, Jack RH, Mak V, Henrik M, Davies EA: The epidemiology and survival of extrapulmonary small cell carcinoma in South East England, 1970-2004. BMC Cancer. 2009, 9:209. 10.1186/1471-2407-9-209

2. Lepage C, Bouvier AM, Faivre J: Endocrine tumours: epidemiology of malignant digestive neuroendocrine tumours. Eur J Endocrinol. 2013, 168:R77-R83. 10.1530/EJE-12-0418

3. Yao JC, Hassan M, Phan A, et al.: One hundred years after “carcinoid”: epidemiology of and prognostic factors for neuroendocrine tumors in 35,825 cases in the United States. J Clin Oncol. 2008, 26:3063-3072. 10.1200/JCO.2007.15.4377 


\section{Cureus}

4. Aslam HM, Iqbal SM, Faizee F, Nida A, Qadir MA: Gastrointestinal neuroendocrine tumor, colon adenocarcinoma, and hyperparathyroidism: an unorthodox synchronism. Cureus. 2018, 10:e3731. 10.7759/cureus.3731

5. Heetfeld M, Chougnet CN, Olsen IH, et al.: Characteristics and treatment of patients with G3 gastroenteropancreatic neuroendocrine neoplasms. Endocr Relat Cancer. 2015, 22:657-664. 10.1530/ERC15-0119

6. Kim JH, Lee SH, Park J, et al.: Extrapulmonary small-cell carcinoma: a single-institution experience. Jpn J Clin Oncol. 2004, 34:250-254. 10.1093/jjco/hyh052

7. Iwasa S, Morizane C, Okusaka T, et al.: Cisplatin and etoposide as first-line chemotherapy for poorly differentiated neuroendocrine carcinoma of the hepatobiliary tract and pancreas. Ipn J Clin Oncol. 2010, 40:313-318. 10.1093/ijco/hyp 173

8. Rinke A, Gress TM: Neuroendocrine cancer, therapeutic strategies in G3 cancers . Digestion. 2017, 95:109114. 10.1159/000454761

9. Olsen IH, Knigge U, Federspiel B, Hansen CP, Skov A, Kjær A, Langer SW: Topotecan monotherapy in heavily pretreated patients with progressive advanced stage neuroendocrine carcinomas. J Cancer. 2014, 5:628-632. 10.7150/jca.9409

10. Paz-Ares L, Dvorkin M, Chen Y, et al.: Durvalumab plus platinum-etoposide versus platinum-etoposide in first-line treatment of extensive-stage small-cell lung cancer (CASPIAN): a randomised, controlled, openlabel, phase 3 trial. Lancet. 2019, 394:1929-1939. 10.1016/S0140-6736(19)32222-6

11. Horn L, Mansfield AS, Szczęsna A, et al.: First-line atezolizumab plus chemotherapy in extensive-stage small-cell lung cancer. N Engl J Med. 2018, 379:2220-2229. 10.1056/NEJMoa1809064

12. Nghiem PT, Bhatia S, Lipson EJ, et al.: PD-1 blockade with pembrolizumab in advanced Merkel-cell carcinoma. N Engl J Med. 2016, 374:2542-2552. 10.1056/NEJMoa1603702 